Many organisations reported the results of field tests on complete information-carrying networks suited to a variety of applications. N. Shimodaira (Nippon Electric Co.) reported the results of field tests on a high bit rate $\left(400 \mathrm{Mb} \mathrm{s}^{-1}\right)$ optical link, the transmission of such high bit rates being made possible by two factors. First, the gradient of the refractive index in the fibre is carefully built up during fabrication, decreasing smoothly from the centre to a constant value in the "cladding". This results in nearly equal group velocities for the various propagating modes which each pulse of light excites. Second, the modes excited by the pulses of light are restricted to those of low order, thus further reducing the total spread. Ga-Al-As semiconductor laser diodes were used to launch pulses into the fibres, and avalanche photodiodes were used to detect them at the receiving end. At the other end of the spectrum W. H. Hackett (Bell Laboratories, Murray Hill) reported the results of tests on short-haul low bit-rate (16 and $32 \mathrm{Mb} \mathrm{s}^{-1}$ ) data links incorporating light-emitting diodes to launch the pulses and PIN photodiodes to detect them.

Information transmission by way of optical links has inherent advantages in environments where high electric fields are present. T. Sekizawa (Fujitsu Laboratories Ltd) reported the results of a cooperative venture which has demonstrated this convincingly in field conditions. They found that the performance of their optical fibre links, which were routed through ducts containing power cables, were not measurably degraded by proximity to high voltages.

Several papers were devoted to splicing techniques. The contribution by P. K. Runge (Bell 'Telephone Laboratories, Holmdel) and W. C. Young (Western Electric) was notable, the connector described being moulded directly to the protective sheath of the optical fibre. The authors quoted losses per connector in the region of $0.4 \mathrm{~dB}$. However it was clear that more work is necessary before a viable splicing system becomes available for general field use.

In the field of fibre fabrication a great deal of effort has been devoted to producing consistently very strong fibres. Both C. Kao (ITT ElectroOptical Products Division) and C. R. Kurkjian (Bell Laboratories, Murray Hill) quoted breaking strengths in excess of $500 \mathrm{k}$.p.s.i. for $125 \mu \mathrm{m}$ diameter fibre by ensuring that surface defects and contamination of the fibres during manufacture were kept to a minimum. Fatigue strengths of fibres under applied stress were also reported which indicated that potential service lives greater than 20 years can be expected for fibre now being produced.

The overall impression gained was that optical communications systems are now a viable proposition for capacity data links. Concerning the future for long haul telecommunications, the question is now how soon?

\section{Short range nuclear forces}

\section{from P. E. Hodgson}

EXPERIMENTS on the photo-proton reaction on oxygen from 100 to $300 \mathrm{MeV}$ by a joint MIT-Glasgow group have recently provided evidence that in this energy range the reaction proceeds through the $\Delta$ (1232) nucleon isobar as an intermediate state, and it is likely that this process is increasingly important at high energies (Phys. Rev. Lett. 38, 8; 1977).

The experiment was carried out by bombarding a beryllium target with a bremmstrahlung beam and measuring the spectrum of the emitted protons. The differential cross sections for the photo-proton reactions on oxygen that leave the residual nucleus in its ground state are shown for several angles as a function of photon energy in Fig. 1.

It is simplest to suppose that the reaction proceeds by a single-step process in which the incident photon interacts with a single proton in the target nucleus and knocks it out. This is indicated diagrammatically in Fig. $2 a$, and

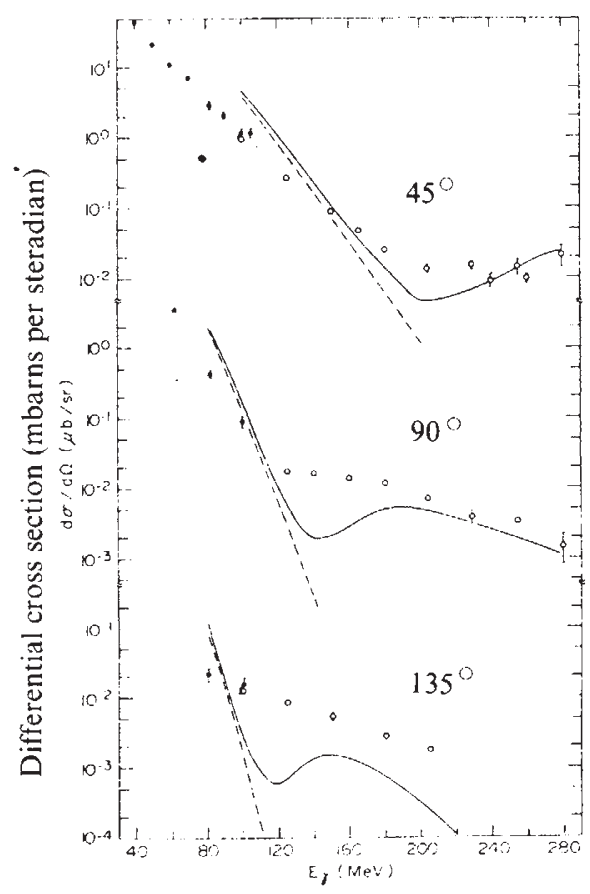

Photon energy
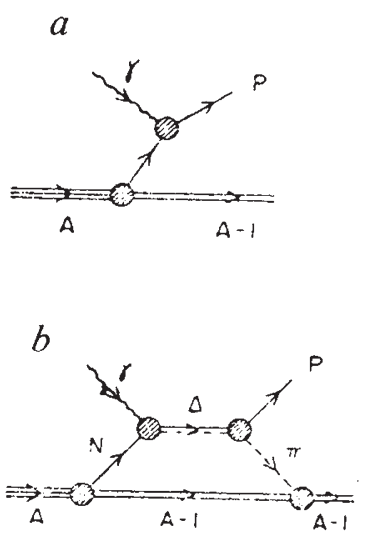

gives the dashed curves in Fig. 1. It is clear that this theory, allowing for uncertainties in the calculations, is in qualitative accord with the data at low energies, but quite fails at higher energies. The calculations are rather rough, since harmonic oscillator wave functions were used, and the distortion of the wave function of the outgoing proton was neglected, but this does not affect the general conclusion.

The experimental cross sections at higher energies are much higher than those given by the one-step process. Such cross sections can be attributed phenomenologically to short-range correlations due to the repulsive core of the nucleon-nucleon interaction or a microscopic model can be made in terms of a two-step process. One such process, involving the $\Delta(1232)$ nucleon isobar excited in an intermediate state, is indicated in Fig. 2b. Calculation with this process gives the full curves in the figure, when added to the results of the calculations of the one-step process already considered. Although there are differences in detail due to the approximations made in the calculation, it is clear that the overall trends are qualitatively given. This provides evidence that the $\Delta$-process can make a major contribution to the photo-proton crosssection in the $100-300 \mathrm{MeV}$ region, and thus gives further insight into the structure of the short-range nuclear forces.

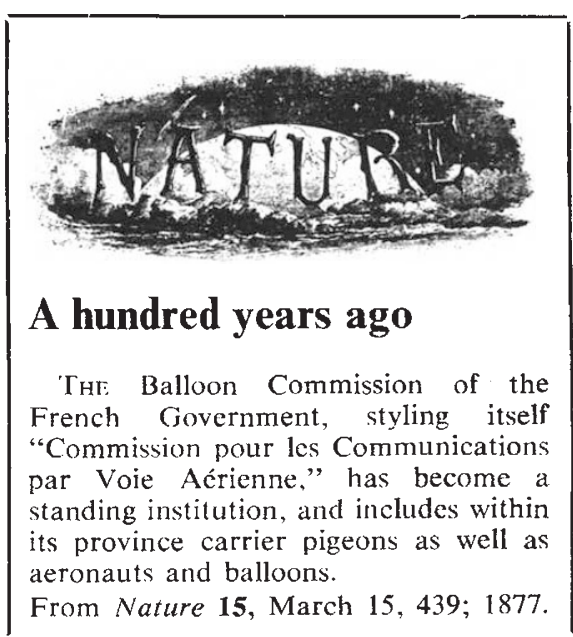

